by the ad hoc Task Force of the ILAE Commission on Therapeutic Strategies. Epilepsia 2010;51(6):1069-77.

2. Freeman JM, Vining EP, Kossoff EH, Pyzik PL, et al. A blinded, crossover study of the efficacy of the ketogenic diet. Epilepsia 2009;50(2):322-5.

3. Bough KJ, Rho JM. Anticonvulsant mechanisms of the ketogenic diet. Epilepsia 2007;48(1):43-58.

4. Kossoff EH, Krauss GL, McGroganJR, Freeman JM. Efficacy of the Atkins diet as therapy for intractable epilepsy. Neurology 2003;61(12):1789-91.

5. Kossoff EH, McGrogan JR, Bluml RM, Pillas DJ, et al. A modified Atkins diet is effective for the treatment of intractable pediatric epilepsy. Epilepsia 2006;47(2):421-4.

6. Sharma S, Sankhyan N, Gulati S, Agarwala A. Use of the modified Atkins diet for treatment of refractory childhood epilepsy: A randomized controlled trial. Epilepsia 2013;54(3):481-6.

7. Neal EG, Chaffe H, Shawartz RH, Lawson MS, et al. The ketogenic diet for the treatment of childhood epilepsy: a randomised trial. Lancet Neurol 2008;7(6):500-6.
8. Kossoff EH, DorwardJL. The Modified Atkins Diet. Epilepsia 2008;49(suppl 8):37-41.

9. Lee PR, Kossoff EH. Dietary treatments for epilepsy: management guidelines for the general practitioner. Epilepsy Behav 2011;21(2):115-21.

10. Kossoff EH. Intractable childhood epilepsy: choosing between the treatments. Semin Pediatr Neurol 2011;18(3):145-9.

11. Miranda MJ, Mortensen M,PovisenJH, Nielsen H, Beniczky S. Danish study of a Modified Atkins diet for medically intractable epilepsy in children: Can we achieve the same results as with the classical ketogenic diet? Seizure 2011;20(2):151-5.

12. Auvin S. Should we routinely use modified Atkins diet instead of regular ketogenic diet to treat children with epilepsy? Seizure 2012;21(4):237-40.

13. Lee PR, Kossoff EH. Dietary treatments for epilepsy: management guidelines for the general practitioner. Epilepsy Behav 2011;21(2):115-21.

\title{
Diecinueve años de vigilancia de enfermedad invasiva neumocócica en un hospital pediátrico de Mendoza, Argentina
}

\author{
Nineteen-years of pneumococcal invasive disease surveillance in a children's \\ hospital in Mendoza, Argentina
}

\author{
Dr. Héctor José Abate ${ }^{a}$ Dra. Andrea Falaschi ${ }^{a}$, Bioq. Laura Balbib y Bioq. Beatriz García ${ }^{b}$
}

\section{RESUMEN}

Estudiamos 537 niños internados en el Hospital Dr. Notti, entre 1993 y 2011, con enfermedad invasiva neumocócica. La mediana de edad fue 19 meses ( $\mathrm{R}=0-192 \mathrm{~m}) ; 34,82 \%$ fueron $<1$ año y $23,46 \%, \geq 60$ meses. Predominaron neumonía con y sin derrame $(48,04 \%)$ y meningitis $(29,05 \%)$, con una letalidad de $6,14 \%$. El 56,86\% de los serotipos identificados fueron 14,5 y 1 . Mostraron sensibilidad a la penicilina el $99,74 \%$ de cepas no meníngeas y a la ceftriaxona, el $98,08 \%$ de cepas meníngeas. Los factores de riesgo en neumonía con derrame se asociaron a la edad $\geq 60$ meses, RR 1,47 (1,06-2,04), p 0,02, serotipos 5, RR $2,57(1,71-3,87)$, p 0,0001 y 1 RR $1,86(1,17-2,96)$, p 0,014 y en

a. Servicio de Infectología.

b. Servicio de Microbiología.

Hospital Pediátrico Dr. Humberto Notti.

Guaymallén. Mendoza. Argentina.

\section{Correspondencia:}

Dr. Abate, Héctor José: hjabate@gmail.com.

Conflicto de intereses: Ninguno que declarar.

Recibido: 31-7-2013

Aceptado: 12-3-2014 las meningitis, principalmente $\mathrm{a}<1$ año, RR 2,35 (1,87-3,06), $\mathrm{p}$ 0,0000 y serotipo 18C, RR 2,19 (1,3-3,7), p 0,024.

Conclusión. El Streptococcus pneumoniae representó un problema importante en menores de un año, en quienes predominó la meningitis y causó más de la mitad de las muertes, y en mayores de 60 meses, en los que prevalecieron neumonías con derrame. La mayoría fueron sensibles a la penicilina y a la ceftriaxona. Palabras clave: enfermedad invasiva neumocócica, serotipos, sensibilidad antibiótica, factores de riesgo.

http://dx.doi.org/10.5546/aap.2014.352

\section{INTRODUCCIÓN}

El Streptococcus pneumoniae (Spn) causa enfermedades inmunoprevenibles de alta mortalidad. ${ }^{1}$ Una revisión de datos en América Latina y el Caribe, entre 1990 y 2006, mostró que entre 12000 y 28000 menores de 5 años podrían morir anualmente a causa de esta enfermedad. ${ }^{2}$

Los serotipos responsables de la mayoría de las enfermedades invasivas neumocócicas (EIN) muestran variaciones geográficas y según el tipo de enfermedad, ${ }^{3}$ además de cambios en la susceptibilidad antimicrobiana, especialmente a la penicilina y a las cefalosporinas de tercera 
generación. ${ }^{4}$ En Argentina, según datos del Sistema de Redes de Vigilancia de los Agentes Bacterianos Responsables de Neumonía y Meningitis (SIREVA), 2012, en menores de 5 años, $79,2 \%$ de las cepas identificadas en meningitis fueron sensibles a la penicilina y el $100 \%$ en cuadros no meníngeos. Para la ceftriaxona, $94,3 \%$ fueron sensibles en meningitis y $5,7 \%$ con sensibilidad intermedia (SI) y, en procesos no meníngeos, $98,5 \%$ fueron sensibles y $1,5 \%$ con SI. ${ }^{5}$

En el año 2012 (Resolución 502/2011), Argentina incorporó la vacuna conjugada neumocócica 13 valente (VCN-13), a partir de los 2 meses de edad. ${ }^{6}$ Conocer la epidemiología de EIN, la distribución de serotipos y la susceptibilidad antimicrobiana es esencial para evaluar el potencial impacto de un programa de vacunación.

\section{OBJETIVOS}

1. Describir las características epidemiológicas de los pacientes internados con EIN.

2. Detectar los factores de riesgo para EIN.

\section{POBLACIÓN Y MÉTODO}

Estudio observacional, analítico y retrospectivo, realizado entre enero de 1993 y diciembre de 2011.

Se seleccionaron todos los pacientes con diagnóstico confirmado de EIN, internados en el Hospital Pediátrico Dr. Humberto Notti de Mendoza. Se definió EIN confirmada al aislamiento de $S p n$ en un fluido normalmente estéril y cuadro clínico compatible.

Se analizaron las historias clínicas, a partir de los registros del Servicio de Microbiología, y se completó una ficha con datos demográficos, formas clínicas de presentación, enfermedad de base, sitio de aislamiento de Spn, susceptibilidad antibiótica, serotipo y letalidad.

Durante todo el estudio, se realizó la serotipificación por el método de Neufeld Quellung, en el Servicio de Bacteriología Clínica INEI-ANLIS Dr. Carlos Malbrán, y la concentración inhibitoria mínima (CIM) para penicilina y ceftriaxona por el método del E Test, y se ajustaron los valores a los puntos de corte del Clinical and Laboratory Standards Institute (CLSI) de 2008. ${ }^{5}$

Las variables cuantitativas se expresaron como mediana y rango, y las cualitativas, en porcentaje.

Se realizó un análisis univariado, con Epidat versión 3.1, para evaluar factores de riesgo para EIN, en el que se informó riesgo relativo (RR), intervalo de confianza del 95\% (IC95\%) y p significativa $<0,05$.

El estudio fue aprobado por el Comité de Ética en Investigación Dr. H. Notti y no se requirió firma de consentimiento informado. Se garantizó confidencialidad de la identidad del paciente.

\section{RESULTADOS}

Se incluyeron 537 niños con EIN, con una proporción media anual de 18,53 casos por 10000 egresos hospitalarios (rango: 7,82/10 000$35,66 / 10$ 000).

En la Tabla 1, se detallan algunas características de la población estudiada.

El 34,82\% (n: 187) eran $\leq 11$ meses; 19,36\% (n: 104), de entre 12 y 23 meses; $10,80 \%$ (n: 58), de entre 24 y 35 meses; 6,70\% (n: 36), de entre 36 y 47 meses; $4,84 \%$ (n: 26), de entre 48 y 59 meses; y $23,46 \%$ (n: 126), $\geq 60$ meses.

Las formas clínicas de presentación por grupo etario se ven en la Figura 1.

Presentaron enfermedad de base el 4,10\% (n: 22) de los niños, distribuidas en síndrome nefrótico: 8; neoplasia: 7; inmunodeficiencia: 2;

TABLA 1. Características de los pacientes internados con diagnóstico de enfermedad invasiva neumocócica

\begin{tabular}{lc} 
Total de pacientes: $\mathbf{5 3 7}$ & \\
Mediana de edad (rango) & 19 meses $(0-192$ meses) \\
Varones & $305(56,8 \%)$ \\
\hline Formas clínicas & $156(29,05 \%)$ \\
Meningitis & $136(25,32 \%)$ \\
Neumonía & $122(22,72 \%)$ \\
Neumonía con derrame & $71(13,22 \%)$ \\
Bacteriemia & $36(6,70 \%)$ \\
Sepsis & $8(1,49 \%)$ \\
Celulitis & $6(1,12 \%)$ \\
Artritis & $2(0,37 \%)$ \\
Peritonitis primaria & \\
\hline Enfermedad de base & $22(4,10 \%)$ \\
Total & 60 meses (de 4 a 195 meses $)$ \\
Mediana de edad (rango) & \\
\hline Letalidad & $33(6,14 \%)$ \\
Global & $18(54,54 \%)$ \\
Por meningitis & $8(24,24 \%)$ \\
Por sepsis & $7(21,21 \%)$ \\
Por neumonía & \\
\hline
\end{tabular}


HIV: 1; implante coclear: 1; fístula de LCR: 1; enfermedad pulmonar crónica: 1; y cardiopatía: 1.

Ningún sujeto registró antecedente de vacunación antineumocócica o EIN intrahospitalaria.

Se serotipificaron 255 cepas $(47,5 \%)$. Los serotipos 14, 5 y 1 representaron el 56,86\% (n: $145)$ del total. EI $88 \%(225 / 255)$ de los serotipos identificados están incluidos en la VCN-13. No se observó circulación de serotipos 3 y 4 . La distribución de serotipos por grupo etario se muestra en la Figura 2.

FIgURA 1. Formas clínicas de presentación según grupo etario

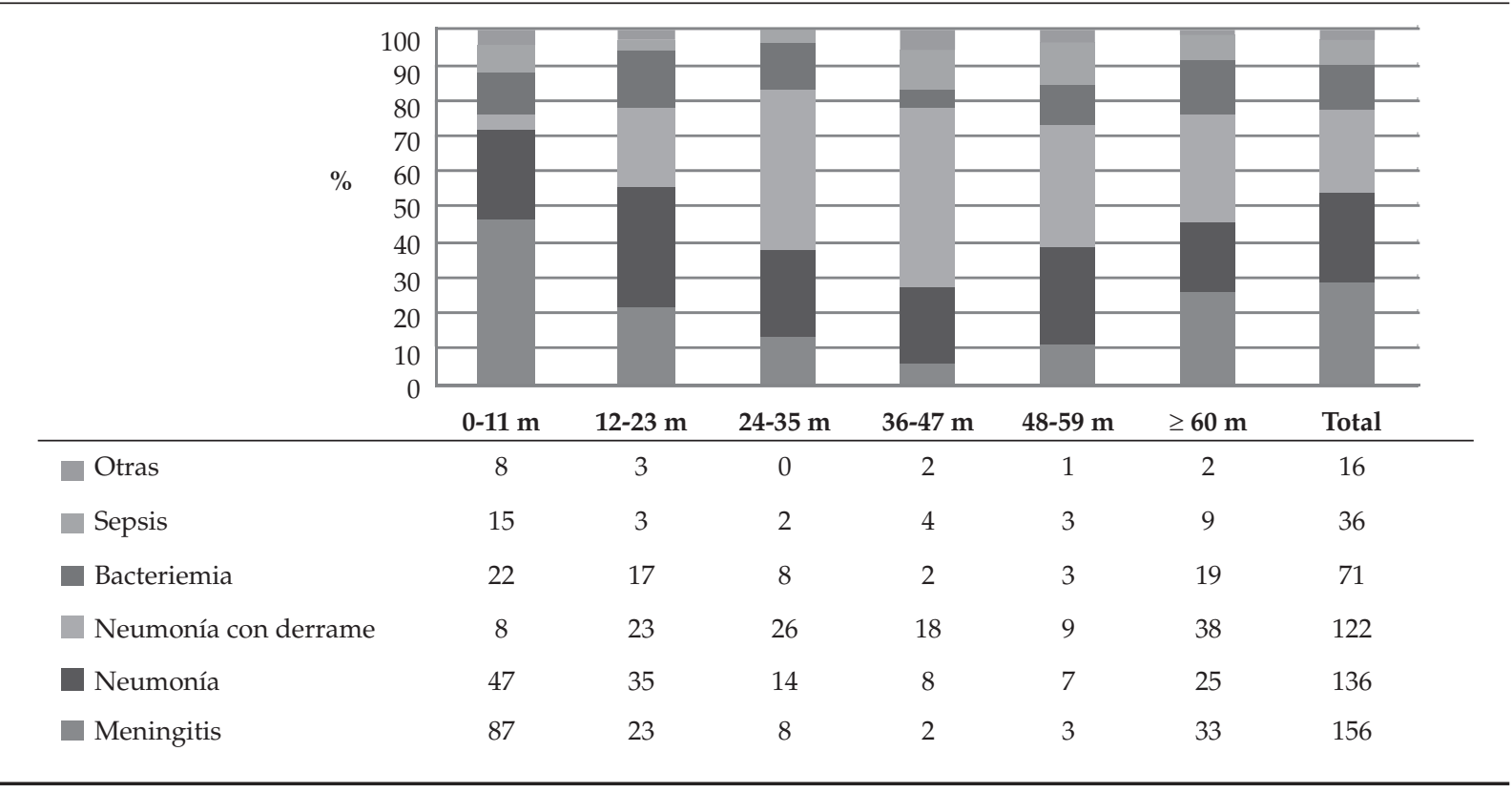

Figura 2. Distribución de serotipos según grupo etario

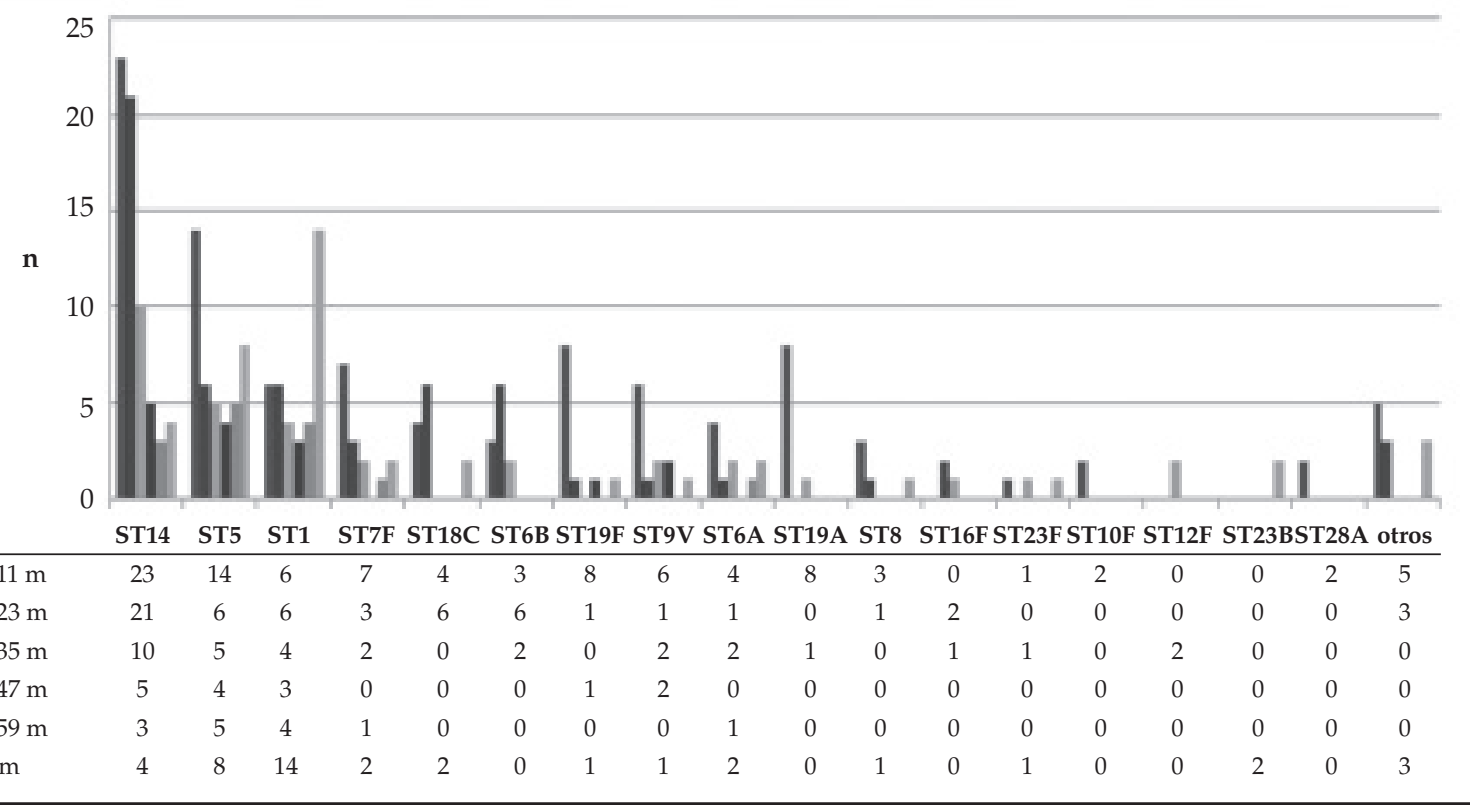

ST: serotipo.

*Otros con un ST: 11, 12, 16A, 18A, 18B, 2, 20, 35F, 42, 7C, 15B. 
En meningitis, resultaron sensibles a la penicilina $89,10 \%$ (n: 139) de las cepas; a la ceftriaxona, $98,08 \%$ (n: 153 ) y con SI, $0,26 \%$ (n: 1 ). En localizaciones no meníngeas, el 99,74\% (n: 380) mostró sensibilidad a la penicilina y $0,26 \%$ (n: 1 ), SI; para ceftriaxona, $0,26 \%$ (n: 1) resultó con SI.

Mostraron resistencia a la penicilina 6,67\% (n: 17) del total de serotipos, repartidos en 14 (n: 8), 6B (n: 3), 19F (n: 2), 18C (n: 1), 9V (n: 1), 23F (n: 1), 23B (n: 1) y SI a la ceftriaxona el 1,18\% (n: 3), el serotipo 14 (n: 2) y 23F (n: 1).

Para el análisis de riesgo relativo para cada forma clínica, según edad, sin ajuste de comorbilidades, véase la Tabla 2.

En los menores de un año, se asociaron significativamente los serotipos 19F: RR 2,01 (IC95\% 1,35-3,0), p: 0,01 y 19A: RR 2,48 (IC95\%
1,86-3,30), p: 0,0021. En $\geq 60$ meses, el serotipo 1: RR 3,05 (1,77-5,26), p: 0,0004. En neumonía con derrame, se asociaron los serotipos 5: RR 2,57 (IC95\% 1,71-3,87), p: 0,0001 y 1: RR 1,86 (IC95\% 1,17-2,96), p: 0,014. En meningitis, el 18C: RR 2,19 (IC95\% 1,3-3,7), p: 0,024.

El riesgo para letalidad se presentó en sepsis: RR 4,45 (IC 95\% 2,16-9,15), p: 0,0008; meningitis: RR 3,46 (IC95\% 1,67-7,16), p: 0,0007; y serotipo 18C: RR 3,37 (IC95\% 1,21-10,54), p: 0,05.

\section{DISCUSIÓN}

Al ser un estudio de base hospitalaria, y teniendo en cuenta que aproximadamente la mitad de los casos de EIN requieren internación, 7,8 probablemente está subestimada la verdadera carga de EIN al no incluir pacientes febriles

TABLA 2. Riesgo relativo para presentar cada forma clínica según edad, sin ajuste de comorbilidades

\begin{tabular}{|c|c|c|c|c|c|}
\hline Forma clínica & Edad & $\mathbf{N}$ & RR & IC (95\%) & $\mathbf{P}$ \\
\hline Meningitis & $<1$ año & 87 & 2,35 & $1,87-3,06$ & 0,0000 \\
\hline \multirow[t]{5}{*}{ (N: 156) } & $<2$ años & 110 & 2,02 & $1,49-2,72$ & 0,000 \\
\hline & $<3$ años & 118 & 1,67 & $1,21-2,3$ & 0,006 \\
\hline & $<4$ años & 120 & 1,34 & $0,97-1,85$ & NS \\
\hline & $<5$ años & 123 & 1,14 & $0,82-1,58$ & NS \\
\hline & $>5$ años & 33 & 0,87 & $0,63-1,21$ & NS \\
\hline Neumonía & $<1$ año & 47 & 0,98 & $0,72-1,34$ & NS \\
\hline \multirow[t]{5}{*}{ (N: 136) } & $<2$ años & 82 & 1,28 & $0,95-1,73$ & NS \\
\hline & $<3$ años & 96 & 1,29 & $0,93-1,98$ & NS \\
\hline & $<4$ años & 104 & 1,28 & $0,95-1,81$ & NS \\
\hline & $<5$ años & 111 & 1,36 & $0,92-2,00$ & NS \\
\hline & $>5$ años & 25 & 0,73 & $0,49-1,07$ & NS \\
\hline Neumonía con derrame & $<1$ año & 8 & 0,13 & $0,06-0,26$ & 0,000 \\
\hline \multirow[t]{5}{*}{ (N: 122) } & $<2$ años & 31 & 0,28 & $0,19-0,41$ & 0,000 \\
\hline & $<3$ años & 57 & 0,47 & $0,34-0,64$ & 0,000 \\
\hline & $<4$ años & 75 & 0,63 & $0,46-0,86$ & 0,004 \\
\hline & $<5$ años & 84 & 0,67 & $0,48-0,93$ & 0,02 \\
\hline & $>5$ años & 38 & 1,47 & $1,06-2,04$ & 0,02 \\
\hline Otras* & $<1$ año & 45 & 1,07 & $0,78-1,48$ & NS \\
\hline \multirow[t]{5}{*}{ (N: 123) } & $<2$ años & 68 & 1,04 & $0,76-1,42$ & NS \\
\hline & $<3$ años & 78 & 0,93 & $0,67-1,28$ & NS \\
\hline & $<4$ años & 86 & 0,91 & $0,66-1,28$ & NS \\
\hline & $<5$ años & 93 & 0,95 & $0,66-1,36$ & NS \\
\hline & > 5 años & 30 & 1,05 & $0,73-1,75$ & NS \\
\hline
\end{tabular}

NS: no significativa. RR: riesgo relativo. IC: intervalo de confianza. 
ambulatorios. Por dicho motivo, predominaron meningitis, neumonía con y sin derrame, y se observó baja frecuencia de bacteriemias. ${ }^{9}$ Esta situación también fue una limitante cuando analizamos el riesgo relativo que implicaba la edad para las diversas formas clínicas de presentación, ya que solo incluimos los niños con enfermedad grave. No obstante, al igual que la bibliografía de América Latina y el Caribe, ${ }^{2,10}$ la mayor incidencia se observó en varones menores de un año, en quienes se asoció el riesgo más alto para meningitis [RR 2,35 (1,87-30,6), p 0,0000] y para serotipos 19F y 19A. Un dato para destacar es que el porcentaje de EIN en el grupo $\geq 60$ meses, como en otros estudios con rangos de edades más amplios, ${ }^{11}$ también fue importante y estuvo asociado significativamente a serotipo $1 \mathrm{y}$ neumonía con derrame.

Si comparamos con otros centros, ${ }^{10,12}$ el porcentaje de sujetos con enfermedad de base fue bajo, posiblemente relacionado con la menor complejidad de los pacientes internados.

Hay que resaltar que la mayoría de las cepas resultaron sensibles a la ceftriaxona, en procesos meníngeos, y a la penicilina, en procesos no meníngeos. Estos datos señalan la importancia de continuar utilizando estos antibióticos en infecciones por Spn.

Consideramos una limitación del estudio no analizar tiempo de internación, evolución y secuelas. No obstante, registramos letalidad, considerada un indicador de gravedad de enfermedad, que mostró un valor intermedio cuando la comparamos con otras regiones ${ }^{7,10,12}$ y un riesgo cuatro veces mayor para sepsis y tres veces para meningitis y serotipo $18 \mathrm{C}$.

Los serotipos 14, 5 y 1 representaron más de la mitad de los serotipos identificados, como en la mayoría de los países latinoamericanos. ${ }^{2,5,9,13} \mathrm{Si}$ bien no figuran en nuestra casuística los serotipos 3 y 4, incluidos en la VCN-13, la mayoría de los serotipos circulantes están incluidos, además de aquellos relacionados con resistencia, excepto el 23B de baja prevalencia. Por consiguiente, es de esperar que la vacuna VCN-13, recientemente introducida en Argentina, logre una reducción importante de las EIN, como fue documentado en otros países que incorporaron vacuna conjugada neumocócica 7 valente (VCN-7) ${ }^{14,15}$ De ahí, la importancia de continuar aportando datos sobre EIN, no solo nacionales sino también regionales, teniendo en cuenta las variaciones que se presentan.

\section{CONCLUSIONES}

La EIN representa un problema importante en niños de Mendoza, especialmente en los menores de un año, en quienes predominó la meningitis, y en los mayores de 60 meses, con mayor número de neumonías con derrame. Un porcentaje pequeño presentó comorbilidad y más de la mitad de las muertes se dieron en los casos con meningitis. La mayoría de los Spn fueron sensibles a la penicilina y a la ceftriaxona.

\section{BIBLIOGRAFÍA}

1. Black RE, Cousens S, Johnson HL, Lawn JE, et al. Global, regional, and national causes of child mortality in 2008: a systematic analysis. Lancet 2010;375(9730):1969-87.

2. Valenzuela MT, O'Loughlin R, De la Hoz F, Gomez E, et al. The burden of pneumococcal disease among Latin American and Caribbean children: review of the evidence. Rev Panam Salud Publica 2009;25(3):270-9.

3. Hausdorff WP, Siber G, Paradiso PR. Geographical differences in invasive pneumococcal disease rates and serotype frequency in young children. Lancet 2001;357(9260):950-2.

4. Cartwright K. Pneumococcal disease in Western Europe: burden of disease, antibiotic resistance and management. Eur J Pediatr 2002;161(4):188-95.

5. Grupo de Trabajo de SIREVA II. Informe regional de SIREVA II 2012: datos por país y por grupos de edad sobre las características de los aislamientos de Streptococcus pneumoniae, Haemophilus influenzae y Neiseriameningitidis en procesos invasores. Washington, DC: OPS 2013. [Acceso: 12 de marzo de 2014]. Disponible en: http:/ / www.paho. org/hq/index.php?option=com_content\&view=category \&layout=blog\&id=3609\&Itemid=3953\&lang=pt.

6. Programa Nacional de Control de Enfermedades Inmunoprevenibles. Ministerio de Salud. Presidencia de la Nación. Introducción de la vacuna conjugada contra neumococo al Calendario Nacional de Inmunizaciones de la República Argentina. Lineamientos técnicos. Argentina, 2011. [Acceso: 12 de marzo de 2014]. Disponible en: http:/ / www.msal.gov.ar/neumococo/descargas/lineamientosneumococo-25-11-2011.pdf.

7. Abarca K, Vergara R, Tassara E, Ibáñez I, et al. Infección neumocócica invasora y neumonía consolidante en lactantes: un año de vigilancia en tres centros hospitalarios chilenos. Rev Chil Infect 2008;25(2):97-103.

8. Lagos R, Muñoz A, Valenzuela M, Heitmann I, Levine MM. Population based surveillance pediatric invasive pneumococcal disease in Santiago, Chile. Pediatr Infect Dis J 2002;21(12):1115-23.

9. Tregnaghi M, Ceballos A, Rüttimann R, Ussher J, et al. Active epidemiologic surveillance of pneumonia and invasive pneumococcal disease in ambulatory and hospitalized infants in Córdoba, Argentina. Pediatr Infect Dis J 2006;25(4):370-2.

10. Ulloa-Gutierrez R, Avila-Aguero M, Herrera M, Herrera $\mathrm{J}$, Arguedas A. Invasive pneumococcal disease in Costa Rican children: a seven year survey. Pediatr Infect Dis J 2003;22(12):1069-74.

11. Guzvinec M, Tesovic G, Tambic-Andrasevic A, ZidovecLepej S, et al. The epidemiology of invasive Streptococcus pneumoniae disease in Croatian children. Med Sci Monit 2008;14(2):59-64. 
12. Pérez GM, Parra A, Casimir L, Mastroiani A, el al. Infecciones invasivas por Streptococcus pneumoniae en un hospital pediátrico de tercer nivel antes de la introducción de la vacuna conjugada. Características clínicas y serotipos involucrados. Arch Argent Pediatr 2013;111(3):202-5.

13. Reijtman V, Fossati S, Hernández C, Sommerfieck P, et al. Serotype distribution of pneumococci isolated from pediatric patients with acute otitis media and invasive infections, and potential coverage of pneumococcal conjugated vaccines. Rev Argent Microbiol 2013;45(1):27-33.

14. Arguedas A, Soley C, Abdelnour A. Prevenar experience. Vaccine 2011;29(Suppl 3);C26-34.

15. Fitzwater SP, Chandran A, Santosham M, Johnson HL. The worldwide impact of the seven-valent pneumococcal conjugate vaccine. Pediatr Infect Dis J 2012;31(5):501-8.

\title{
Archivos hace 75 años
}

Año $X$

Agosto de 1939

Tomo XII, N•2

\section{ARCHIVOS ARgENTINOS DE PEDIATRÍA} PUBLIOACIÓN MENSUAL

(Organo Oficial de la Sociedad Argentina de Pediatría)

\section{Gangrena simétrica de las extremidades en un recién nacido $\left.{ }^{*}\right)$ \\ por los doctores}

\author{
Prof, R. Cíbils Aguirre, J. R. Calcarami y A. Lucero Funes
}

Hemos creído oportuno publicar "in extenso" esta curiosa observación clínica, presentada ya en resumen a la Sociedad de Pediatría en 1938, por la coincidencia de aparecer recientemente una serie de comunicaciones sobre gangrenas simétricas y gangrenas espontáneas en el lactante.

Así Loubat, Villar y Cuzac, estudian el año pasado un caso de gangrena simétrica de las extremidades en un niño de pocos meses, que llega a la eliminación espontánea de los últimos cuatro dedos de cada mano.

Luego, Barbé, ante la Sociedad de Pediatría de París, en enero de este año, presenta la observación de un niño de 18 días, con gangrena simétrica de cuatro dedos de la mano izquierda y de dos dedos de la mano derecha.

Y luego, Huber, Florand y Payet, ante la misma Sociedad, comentan el 28 de febrero próximo pasado, una gangrena espontánea y mutilante de los dedos de ambas manos en un niño de tres semanas.

(*) Presentado a la Sociedad Argentina de Pediatría, en la reunión del 14 de junio de 1938 .

Texto completo en versión electrónica 